Research Paper

\title{
Coccomyxa Gloeobotrydiformis Improves Learning and Memory in Intrinsic Aging Rats
}

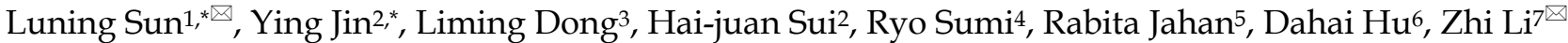 \\ 1. Department of Pathophysiology, College of Basic Medical Science, China Medical University; \\ 2. Department of Pharmacology, Liaoning Medical University; \\ 3. Department of Urology, the Forth Affiliated Hospital, China Medical University; \\ 4. Tanaka Memorial Laboratory, Nikken Sohonsha Corporation; \\ 5. International Education School, China Medical University; \\ 6. Department of Statistics and Finance, School of Management, University of Science and Technology of China; \\ 7. Department of Ethnopharmacology, School of Pharmaceutical Sciences, China Medical University \\ ${ }^{*}$ Luning Sun and Ying Jin equally contributed at this work.
}

$凶$ Corresponding authors: Zhi Li and Luning Sun. E-mail: lizhilele@hotmail.com; lnsun@mail.cmu.edu.cn.

๑ 2015 Ivyspring International Publisher. Reproduction is permitted for personal, noncommercial use, provided that the article is in whole, unmodified, and properly cited. See http://ivyspring.com/terms for terms and conditions.

Received: 2014.10.20; Accepted: 2015.04.23; Published: 2015.05.30

\begin{abstract}
Declining in learning and memory is one of the most common and prominent problems during the aging process. Neurotransmitter changes, oxidative stress, mitochondrial dysfunction and abnormal signal transduction were considered to participate in this process. In the present study, we examined the effects of Coccomyxa gloeobotrydiformis (CGD) on learning and memory ability of intrinsic aging rats. As a result, CGD treated $(50 \mathrm{mg} / \mathrm{kg} \cdot \mathrm{d}$ or $100 \mathrm{mg} / \mathrm{kg} \cdot \mathrm{d}$ for a duration of 8 weeks) 22-month-old male rats, which have shown significant improvement on learning and spatial memory ability compared with control, which was evidently revealed in both the hidden platform tasks and probe trials. The following immunohistochemistry and Western blot experiments suggested that CGD could increase the content of Ach and thereby improve the function of the cholinergic neurons in the hippocampus, and therefore also improving learning and memory ability of the aged rats by acting as an anti-inflammatory agent. The effects of CGD on learning and memory might also have an association with the ERK/CREB signalling. The results above suggest that the naturally made drug CGD may have several great benefit as a multi-target drug in the process of prevention and/or treatment of age-dependent cognitive decline and aging process.
\end{abstract}

Key words: Coccomyxa gloeobotrydifomemory; aging; hippocampus; cholinergic neurons; inflammation; ERK/CREB signalling

\section{Introduction}

The aging of population has been a growing problem in the worldwide, and aging society will be the destination of all countries. In Japan, the population over the age of 65 sums up to more than $20 \%$ of the total amount, which is the highest in the world and will continue to rise ${ }^{[1]}$. In the United States, individuals age 65 and older has increased by $18 \%$ just within the last decade and have estimated to become 79 million by the year 2040, making up $20 \%$ of the total U.S. population [2]. China has the largest popula- tion of elderly citizens in the world, and statistics from the China National Committee on Aging (CNCA) show that the proportion of people older than 60 years has reached to $14.8 \%$ [3]. During the natural aging process, several age-related diseases appear, among which the declining of learning and memory ability is one of the most common and prominent problems, and caring for the elderly with dementia has become a major burden for the family in our society. Therefore, it is crucial for us to develop a medication that will 
help improve learning and memory abilities of the elderly in our communities, and thereby, help prevent the process of dementia. It is well established that oxidative stress is associated with the pathogenesis of several age-related diseases [4] [5] [6] [7], and the presence of low-grade inflammation is a common hallmark of the aging process [8]. The inflammation in the brain plays a key role in the progression of age-related cognitive dysfunction, and increased proinflammatory cytokine IL-1 $\beta$ concentration in cortical and hippocampal tissue was observed by Martin et al [9]. Inflammasomal activation triggered by the proinflammatory factors will cause multiple morphological and neurochemical changes associated with the appearance of declined learning and memory abilities. Recent studies have revealed that mitochondria are crucial regulators of inflammasome activation [10]. The mitochondrial respiratory chain is the major cellular source of ROS (reactive oxygen species) production, and increased ROS could induce the expression and secretion of interleukin-1 $\beta^{[11]}$. Therefore, mitochondrial dysfunction coupled with the aging process may increase the electron leakage and then increase ROS production and stimulates inflammatory responses in the brains.

Our previous study indicated that Coccomyxa gloeobotrydiformis (CGD) treatment could both maintain mitochondrial membrane potential and decrease mitochondrial swelling. In addition, it also prevents oxidative damage in the hippocampus [12]. CGD is a species of green algae, and the data obtained showed a copious amount of a-linolenic acid (ALA), approximately $24 \%$. Besides its antioxidant capacity, ALA also protected hippocampal cells from calcium buffering dysfunction and cell death [13]. Hence, this might be a favorable drug worthy of further investigation for its properties against age-related neurodegenerative alterations, considering its anti-oxidant and neuroprotective effects.

In this study, we aim to observe the therapeutic effects of CGD on age-related deficits in learning and memory in the intrinsic aging rat model. Furthermore, we discuss the underlying mechanisms of the drug and its effects on the specimen.

\section{Materials and methods}

The Institutional Animal Care and Use Committee approved this animal study. All procedures in this study were carried out in accordance with the recommendations in the guide for the care and use of laboratory animals of China Medical University. All surgeries were performed under anesthesia, and all efforts were made to minimize pain and suffering for the animals.

\section{Animals}

The intrinsic aging rat model was used. Young (3 months old) or aged (22 months old) male Sprague-Dawley rats (Experimental Animal Center of Liaoning Medical University) were housed at an ambient temperature of $22-23{ }^{\circ} \mathrm{C}$ under a 12-h light-dark schedule and were maintained under veterinary supervision throughout the study. Aged rats were divided into three groups ( $\mathrm{n}=15$ for each group). Two groups were fed on a CGD diet $(50 \mathrm{mg} / \mathrm{kg} \cdot \mathrm{d}$ and 100 $\mathrm{mg} / \mathrm{kg} \cdot \mathrm{d}$ respectively) for 8 weeks from 22 months of age. The control group received standard laboratory chow. The young rats were divided into the same style of groups. Administration of CGD showed no effect on animal weight or their general condition throughout this period.

\section{Morris water maze test}

Rats were tested in a Morris water maze (MWM; $120 \mathrm{~cm}$ diameter, $50 \mathrm{~cm}$ high, $21^{\circ} \mathrm{C} \pm 1^{\circ} \mathrm{C}$ ), and milk powder was added to prevent visibility of the platform in the pool. A circular target platform was hidden $2 \mathrm{~cm}$ below the water surface in a fixed location in one of four quadrants halfway between the wall and the middle of pool throughout the experiments. During training, animals were required to locate the submerged platform by using distal cues. They were tested for two trials per day for 5 consecutive days. Rats were released from one of four possible starting points and allowed to search the platform for 60s. The rats that could not find the platform within 60 s were guided to the platform and were allowed to remain on the platform for 20s. The time to reach the platform was recorded using a video camera and were fed to an electronic imaging system. During $6^{\text {th }}$ and $7^{\text {th }}$ days, the rats were tested for four trials per day. Training trials on day 7 were followed by a probe trial. For this, the platform was removed and the searching time in the previously platform- containing quadrant was measured over $60 \mathrm{~s}$. This provides a measurement for the retention of spatial memory and indicates whether a spatial strategy was used during hidden platform training.

\section{Tissue processing}

After the Morris water maze experiment, 7 or 8 animals were chosen from each group to be decapitated and their brains were rapidly removed for the tissue sample. Hippocampus tissues were isolated and sliced (500 $\mu \mathrm{m}$ thick) on dry ice. The CA1 and CA3 regions were microdissected under anatomical microscope and frozen at $-80{ }^{\circ} \mathrm{C}$. Then the remaining animals in each group were anesthetized (chloral hydrate, $300 \mathrm{mg} / \mathrm{kg}$ ) and perfused transcardially with $0.1 \mathrm{M}$ PBS, followed by $4 \%$ paraformaldelyde solution 
at $4^{\circ} \mathrm{C}$. Brains were blocked in the coronal plane, post-fixed for $24 \mathrm{~h}$, and embedded in paraffin. Serial coronal sections ( $8 \mu \mathrm{m}$ thickness) were taken from hippocampus (from Bregma -3.14 mm to Bregma -3.8 $\mathrm{mm}$ ) for immunohistochemical staining.

\section{Immunohistochemical staining}

The sections of hippocampus were incubated with polyclonal antibody against glial fibrillary acidic protein (GFAP; Santa Cruz, CA, USA; 1:100 in TBS), eNOS (Santa Cruz, CA, USA; 1:100 in TBS) or TrkA (Abcam, USA; 1:400 in TBS) and ChAT (Abcam USA; 1:400 in TBS) antibodies overnight at $4^{\circ} \mathrm{C}$, and followed by secondary antibody at $37{ }^{\circ} \mathrm{C}$ for $1 \mathrm{~h}$. The GFAP, eNOS, TrkA and ChAT-positive cells were detected by SABC and DAB kit. All sections through the medial septal nucleus and vertical diagonal band nucleus were saved, starting from $1.6 \mathrm{~mm}$ anterior to bregma through $0.3 \mathrm{~mm}$ posterior to bregma. The one-in-twenty series of histological sections $(160 \mu \mathrm{m}$ spacing) were immunostained for ChAT (total $12 \mathrm{sec}-$ tions) and TrkA (total 12 sections), respectively. Tissue sections from groups were nicked at a predetermined place on the hippocampus (for later identification) and then collected in the same vial and stained free-floating under identical conditions.

\section{Western blot analysis}

Protein lysates of hippocampal CA1, CA3 region or basal forebrain were subjected to $12 \%$ SDS-PAGE and transferred onto nitrocellulose membrane, respectively. After blocking with $5 \%$ skim milk, the membranes were incubated overnight at $4^{\circ} \mathrm{C}$ with their respective primary antibodies [ IL-1 $\beta, 1: 500$, Santa Cruz, U.S.A; ChAT, TrkA, eNOS, phospho-ERK1/2(Thr202/Tyr204), ERK, phospho-CREB(Ser133) and CREB, 1:1000, Cell signaling, U.S.A]. The membranes were then incubated with appropriate secondary horseradish peroxidase-conjugated anti-rabbit or anti-mouse immunoglobulin $\mathrm{G}(\mathrm{IgG})$ and anti-biotin antibody (1:2000, Cell signaling, U.S.A) for $1 \mathrm{~h}$ at room temperature. The blots were detected using the enhanced chemiluminescence (ECL, Amersham, UK) reaction, and the protein bands were quantified using Visionworks 6.3.3 (UVP, USA).

\section{Determination of nitrite oxide (NO)}

Nitrite is a stable end product of $\mathrm{NO}$, and its concentration was determined by the Griess method. Brain was homogenized in four volumes of $30 \mathrm{mM}$ Tris- $\mathrm{HCl}$ buffer, $\mathrm{pH}$ 6.8, containing 5 mM EDTA, 250 $\mathrm{mM}$ sucrose, $30 \mathrm{mM} \mathrm{KCl}, 2 \% \beta$-mercaptoethanol, PMSF $(100 \mu \mathrm{g} / \mathrm{ml})$, benzamidine $(5 \mu \mathrm{g} / \mathrm{ml})$, aprotinin $(2 \mu \mathrm{g} / \mathrm{ml})$ and leupeptin $(2 \mu \mathrm{g} / \mathrm{ml})$, and then centri- fuged $\left(12,000 \times g, 4^{\circ} \mathrm{C}, 15 \mathrm{~min}\right)$. Protein concentrations were determined by the Coomassie blue dye-binding method using bovine serum albumin as standard. Aliquots $(1.0 \mathrm{ml})$ of the homogenates were removed and diluted with $1.0 \mathrm{ml}$ of Griess reagent (1\% sulphanilamide, $2 \%$ phosphoric acid and $0.1 \%$ naphthyl ethylene diamine dihydrochloride). The absorbance of the chromophore formed during diazotization of the nitrite with sulphanilamide and subsequent coupling with naphthylethelene diamine was measured at 545 nm. Appropriate blanks and controls were run in parallel.

\section{Statistical analysis}

All data were analyzed using either Student's t-test or a one-way analysis of variance (ANOVA) followed by post hoc Student-Newman-Keuls test. Data are expressed as mean \pm S.E.M. (Standard error of the mean) and deemed statistically significant when $P<0.05$.

\section{Results}

\section{CGD improved the spatial learning and memory ability in aged rats}

We examined the effects of CGD on learning and memory of both aged and young rats for the hidden platform water maze task. Across 5 days' training, the mean escape latencies (MEL) decreased in both young and aged rats, but the aged rats exhibited significantly higher MEL than the young ones on day 3, 4, and 5 (data not shown). After treated with CGD $(50 \mathrm{mg} / \mathrm{kg}$ or $100 \mathrm{mg} / \mathrm{kg}$ ), the aged rats reached the platform faster than the control of same age on day 3, 4, and 5, and their MEL were similar to those of the young ones (Fig 1A). CGD treatment showed no significant effects on the MEL of the young rats (data not shown). During the probe trial, the young rats spent significantly more time in the target quadrant than in other ones (data not shown), but no significant difference was found in the aged rats. After treated with CGD, the aged rats showed a longer search time in the target quadrant than in other ones $(P<0.05$; ANOVA), and no similar effects were found on the young rats (Fig 1B).

\section{CGD increased choline acetyltransferase (ChAT) and tropomyosin receptor kinase A (TrkA) expression in the hippocampus of aged rats}

ChAT is the crucial enzyme during the acetyl choline (Ach) synthesis, which is found in high concentration in cholinergic neurons. So ChAT is often used as a marker for cholinergic neuron. The concentration of ChAT is remarkably reduced in age related memory impairments ${ }^{[14]}$. Nerve growth factor (NGF) 
can increase the ChAT expression and prevent the cholinergic neuronatrophy [15]. NGF selectively binds to its high affinity receptor TrkA, the downregulation of which is associated with cognitive decline in Alzheimer's disease [16]. In this study, we found that the expression of ChAT in the hippocampal CA1 region of aged rats was significantly increased after being treated with CGD (Fig 2). The other major finding of this part is that CGD significantly increased TrkA protein expression in hippocampal CA3 region (Fig.3 A, B) and basal forebrain (BF) (Fig.3 C, D) , compared with the aged control rats. The strongest intensity in TrkA immunoreactivity was seen in CA3 region of CGD treated aged rats, which was verified by Western blotting (Fig 3G). But no similar effects were found in CA1 region (Fig.3 E, F).

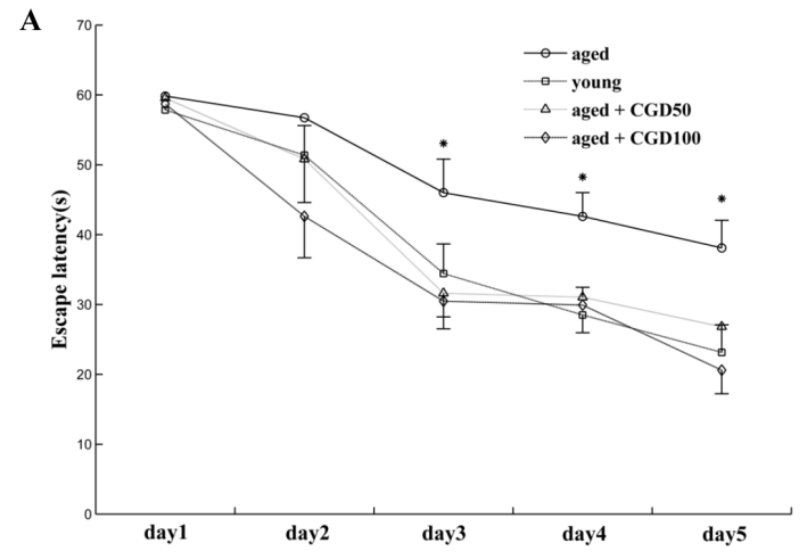

B

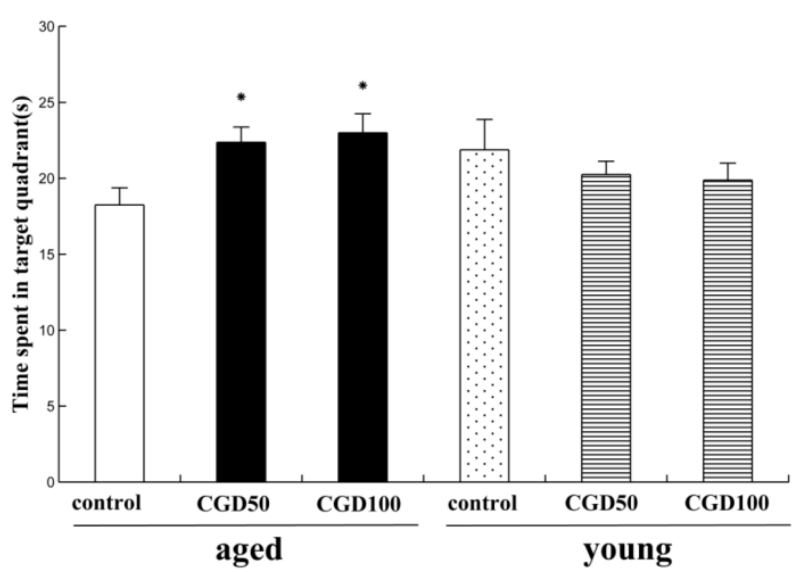

Fig.1. Influence of CGD on Morris water maze performance in the aged rat. A. The escape latency of Morris water maze analysis during the whole training sessions. $B$. The time spent in the target quadrant in the probe trial. Mean \pm SEM $n=15$. ${ }^{*} P<0.05$, compared with the aged control rats. CGD50: $50 \mathrm{mg} / \mathrm{kg}$ CGD treated group; CGD100: $100 \mathrm{mg} / \mathrm{kg}$ CGD treated group.
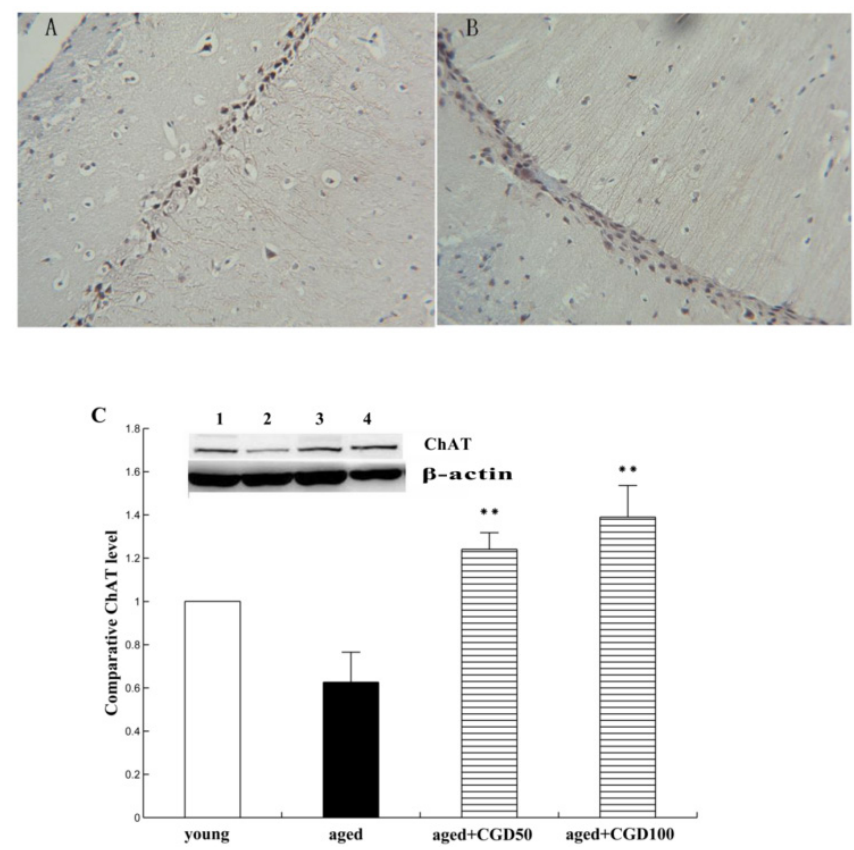

Fig.2. Influence of CGD on ChAT expression in the hippocampus. A. ChAT-positive immunostaining in hippocampal CAl region of control. B. ChAT-positive immunostaining in hippocampal CA1 region of CGD treated group. Magnification: $\times 400$. C. Western blotting of ChAT expression in hippocampal CA1 region. Mean $\pm \mathrm{SEM}$ $n=4$. ${ }^{*} P<0.01$; compared with aged-control rats.

\section{CGD inhibited inflammatory reactions in the hippocampus of aged rats}

The systemic inflammation promotes age-related degenerative changes. Astrocytic reaction was thought to be responsible for the increase in the proinflammatory cytokine. Astrocytes were visualized by means of immunohistochemistry for GFAP, a specific marker of astrocytes. In aged hippocampus, we found the marked activation of astrocytes, which was characterized by the long, thick branching of the cells. After treated with CGD at a daily dose of $100 \mathrm{mg} / \mathrm{kg}$ for 8 weeks, these morphological changes were attenuated significantly (Fig 4 A, B). As shown in Fig 4C, the expression of proinflammatory cytokine IL-1 $\beta$ significantly increased in the hippocampus of aged rats, which was downregulated by CGD. But CGD treatment did not affect IL-1 $\beta$ expression in the young rats (data not shown). To further investigate the anti-inflamatory effects of CGD, we observed the activation of astrocytes in hippocampus. The astrocytes are the major components of the inflamatory reactions in the central nervous system, and the reactive astrocytes contribute to this process by releasing some cytokines such as IL-1 $\beta$. 

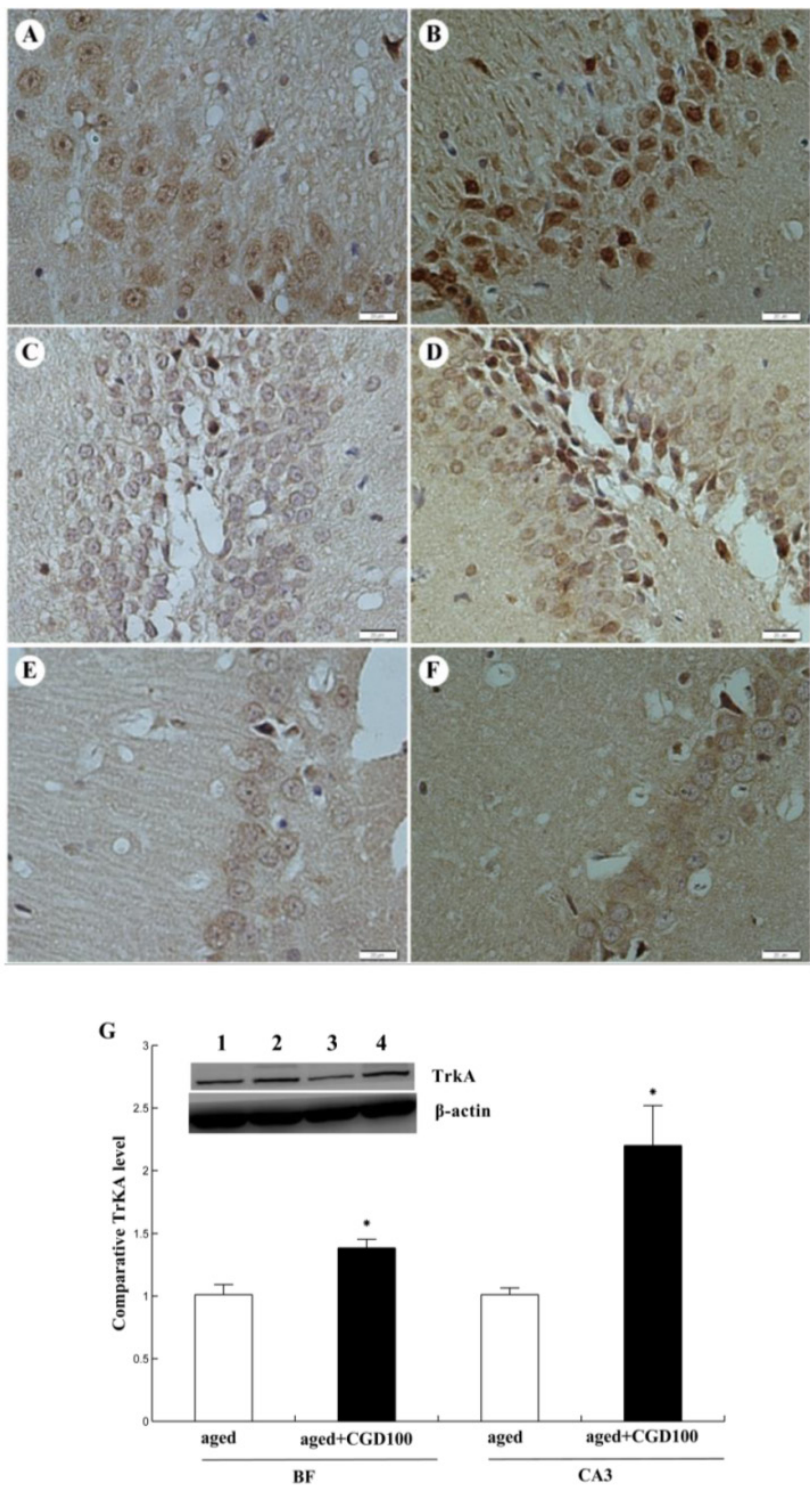

Fig.3. Influence of CGD on TrkA protein expression in hippocampus of aged rats. A. TrkA immunostaining in hippocampal CA3 region of control group. B. TrkA immunostaining in hippocampal CA3 region of CGD treated group. C. TrkA immunostaining in granule cells of the basal forebrain (BF) of control group. D. TrkA immunostaining in granule cells of the dentate gyrus of CGD treated group. E. TrkA immunostaining in hippocampal CAl region of control group. F. TrkA immunostaining in hippocampal CA1 region of CGD treated group. Magnification: $\times 400$. G. Western blotting of TrkA protein expression in hippocampal CA3 region and BF. Mean \pm SEM $n=4 * P<0.05$; compared with aged-control rats.

\section{CGD increased the endothelial nitric oxide synthase (eNOS) expression and nitric oxide (NO) production in the hippocampus of the aged rats}

NO is a very important cellular signal molecule during the neurotransmission, and its synthesis is regulated by nitric oxide synthase including neural nitric oxide synthase (nNOS), endothelial nitric oxide synthase (eNOS) and induced nitric oxide synthase (iNOS). eNOS is a key mediator in anti-inflammatory action, and iNOS is a pro-inflammatory mediator which can trigger the synthesis of some cytokines ${ }^{[17]}$.
In this study, eNOS protein expression was significantly increased in hippocampal CA1 and CA3 regions of CGD treated aged rats, especially CA3 region, compared to the aged control group (Fig 5A-E). eNOS immunoreactivity in the perikarya and dendritic processes of CA1 pyramidal cells was more intense than in the control and the staining was continuous and extensive along the dendritic processes of CA1 pyramidal cells. However, no significant change was observed in cerebral cortex (data not shown). Consistent with the change of eNOS protein expression, the NO production was significantly increased in hippocampal CA1 and CA3 areas of CGD treated aged rats, compared with the aged control rats. No significant change was observed in cortex and basal forebrain after CGD treatment (Fig.5F).

\section{CGD increased the phosphorylation of ERK1 and CREB}

The activation of ERK-CREB signaling pathway may improve the spatial working memory ${ }^{[18]}$. To further explore the signaling mechanisms underlying the protective effect of CGD on age-related learning and memory deficits, extracellular signal-regulated kinase $1 / 2(E R K 1 / 2)$ and CREB signaling were analyzed. Fig 6A showed that protein level of phospho-ERK1 was significantly reduced in the hippocampal CA1 of aged rats compared with the young ones $(P$ $<0.01$;ANOVA), but after treated with CGD, the phospho-ERK1 level in the hippocampal CA1 of the aged rats increased and were even greater than those in tissue from young rats $(P<0.01$; ANOVA). Fig. 6B showed the protein level of phospho-CREB; the evidence from blot and mean data indicated that CREB phosphorylation was significantly increased in hippocampal CA1 areas prepared from $100 \mathrm{mg} / \mathrm{kg}$ CGD-treated aged rats, compared with the aged control rats $(P<0.05 ;$ ANOVA). No difference in total ERK1 and CREB were observed between CGD treated and control groups.

\section{Discussion}

Learning and memory impairments are common manifestations of brain aging in both humans and laboratory animals [19]. Abnormal cholinergic system, oxidative stress, mitochondrial dysfunction and abnormal signal transduction were considered to participate in this process [20], but there is still no effective treatment by now. The possibility that this problem could be reversible and restored by drug treatment offers great hope for the rapidly growing aging population, and it will be ideal for the candidate drug to be naturally produced with very minimal side effects. The present study showed that CGD could improve learning and memory in intrinsic aging rats, which 
was evident in both the hidden platform tasks and probe trials. Then the effects of CGD on the neurotransmitters, inflammatory factor and the related
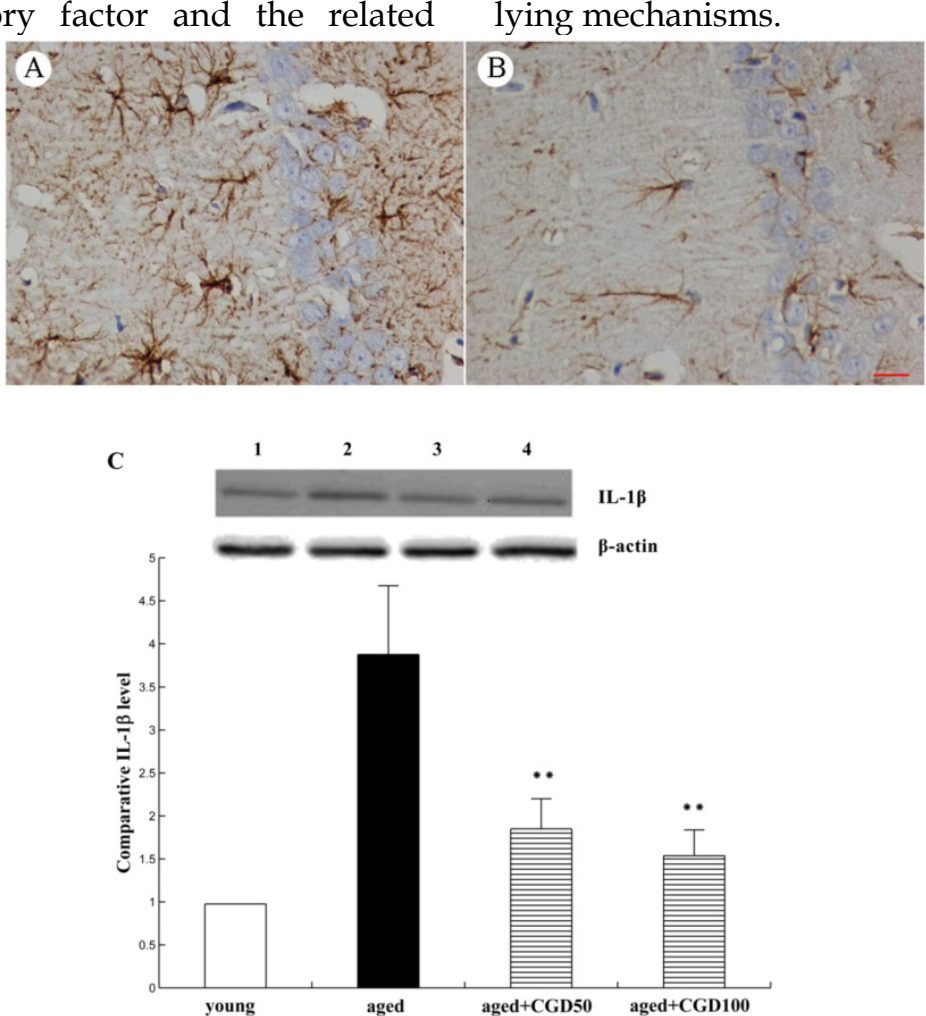

Fig.4. Influence of CGD on inflammatory reactions in the hippocampus. A. GFAP immunostaining of astrocytes in hippocampal CA1 region of aged control rats. B. GFAP immunostaining of astrocytes in hippocampal CAl region of CGD-treated aged rats. Magnification: $\times 400$. $C$. IL- $1 \beta$ expression in hippocampal CAl region. Mean \pm SEM $n=4$ * $P<0.01$; compared with aged control rats.

Fig.5. Influence of CGD on eNOS expression and NO production in hippocampus. A. eNOS immunohistochemistry in hippocampal $\mathrm{CAl}$ region of the aged control. $\mathrm{B}$. eNOS immunohistochemistry in hippocampal CAl region of the CGD-treated aged rats. C. eNOS immunohistochemistry in hippocampal $\mathrm{CA} 3$ region of the aged control. D. eNOS immunohistochemistry in hippocampal CA3 region of the CGD-treated aged rats. Magnification: $\times 400$. E. Western blotting of eNOS in hippocampal CA1 and CA3 regions. Mean \pm SEM $n=4 ;{ }^{*} P<0.05$; ${ }^{*} P<0.01$; compared with aged-control rats. F. Nitrite levels in hippocampal CA1, hippocampal CA3, Basal forebrain (BF), and temporal cortex (TC). Mean $\pm S E M n=8$. ${ }^{*} P<0.05$; ${ }^{*} \mathrm{P}<0.01$; compared with aged control rats. signal transducers in the hippocampus of the aged rats were further investigated to explain the underlying mechanisms.
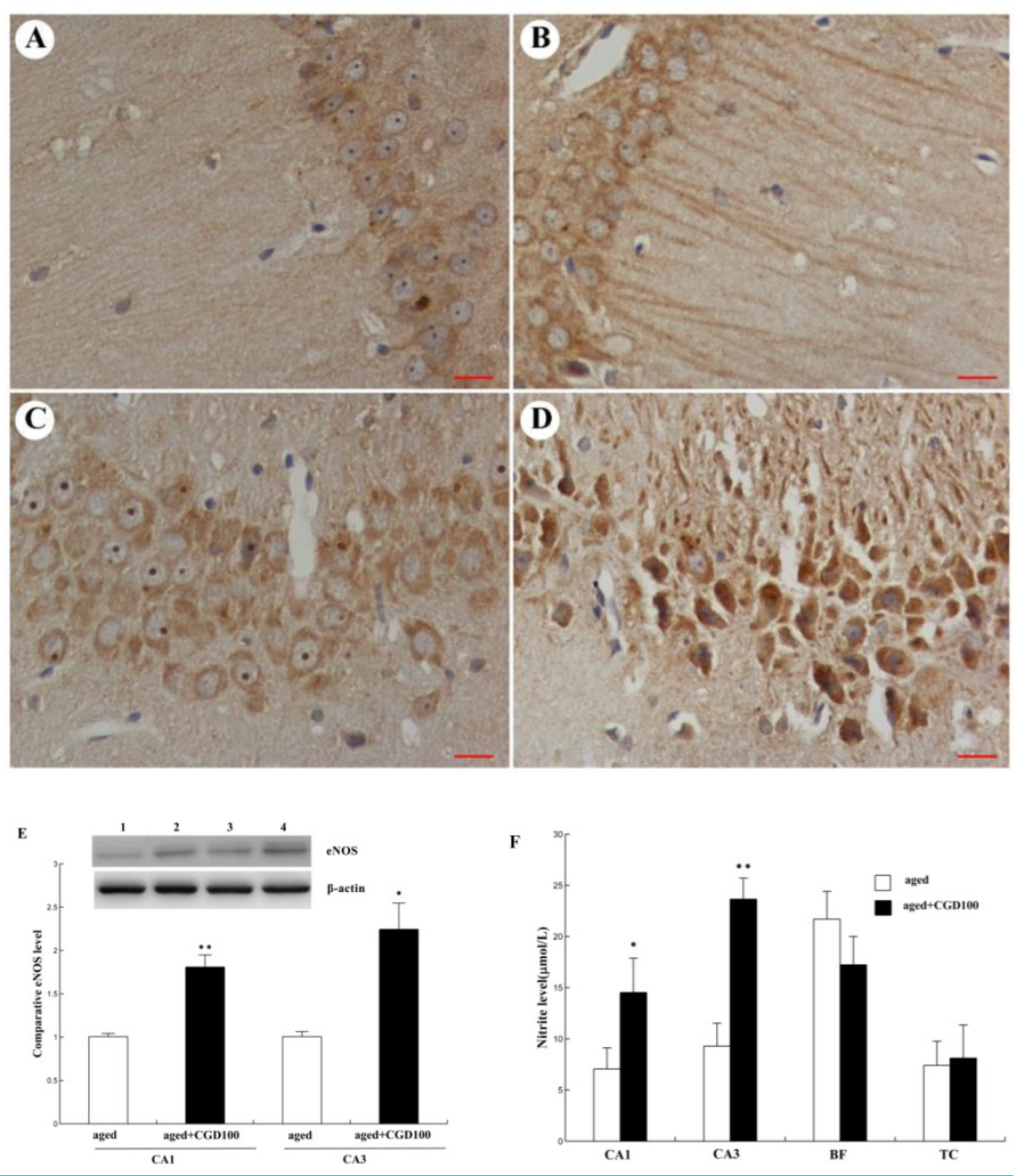

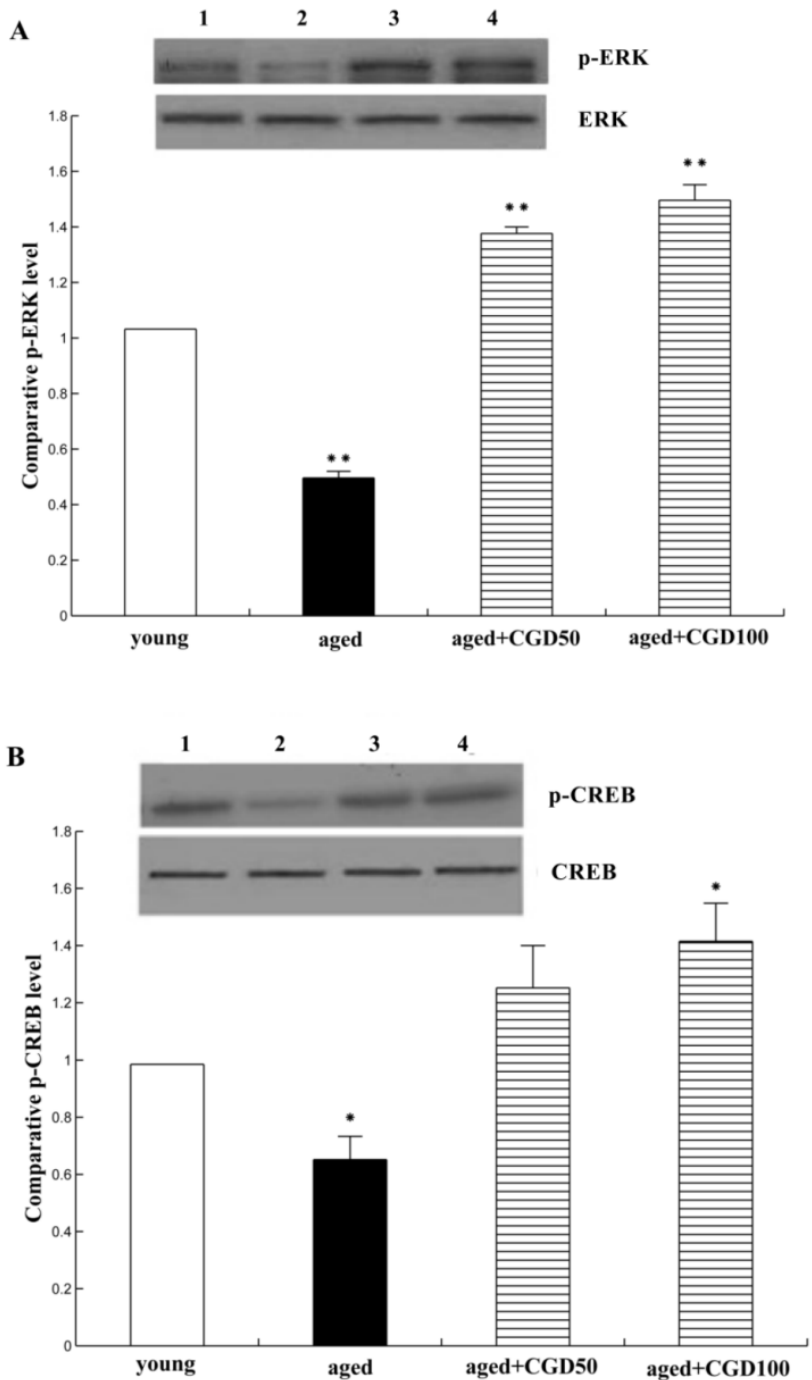

Fig.6. Influence of CGD on the phosphorylation of ERK1 and CREB. A. Western blotting of phospho-ERK1 in hippocampal CAl region. B. Western blotting of phospho-CREB in hippocampal CAl region. Mean \pm SEM $n=4$. ${ }^{*} P<0.05 ;{ }^{* *} P<0.01$; compared with aged-control rats.

The functions of cholinergic system are closely related to learning and memory. NGF plays a role in maintenance of the cholinergic neurons and morphological phenotype ${ }^{[21]}$. It binds to its high-affinity receptor TrkA and causes the dimerization, followed by autophosphorylation of multiple tyrosine residues, which in turn initiates signal transduction events that generally promote the survival of cholinergic neurons. In neurodegenerative processes, loss of TrkA density correlates with neuronal atrophy and precedes neuronal death [22]. In this study, ChAT was used as a marker for cholinergic neuron. We found that CGD significantly increased the intensity of ChAT-immunoreactive fibers in hippocampus of aged rats. Since the expression of NGF did not change, we observed the alteration of its receptor TrkA. As a result, TrkA expression had significantly increased in DG (dentate gyrus) and CA3 but not in CA1 hippo- campal regions after treated with CGD. Shing et al proposed the possibility of dissociation between functions of hippocampal regions. For example, reduced CA3/DG was involved in false recognition memory among older adults and decreased CA1 volume might induce general cognitive deficit [23]. Therefore, we proposed that CGD might improve the function of the cholinergic neurons in the hippocampus DG and CA3 of the aged animals through potentiating NGF/TrkA signaling. Further studies are required to clarify the mechanisms that involved in the hippocampus CA1.

As mentioned earlier, inflammation in the brain plays key roles in the progression of age-related cognitive dysfunction. It has been reported that proinflammatory cytokine IL-1 $\beta$ was markedly increased in hippocampus of aged rats [24]. IL-1 $\beta$ has also been shown to trigger cell death in primary cultures of human fetal neurons [25], so it might be the trigger that induces the age-related changes in the brain and therefore be responsible for the deficit in learning and memory. In addition, astrocytes have been reported to play an important role in inducing inflammatory changes. The age-related astrogliosis and astrocytosis were responsible for the increase in the proinflammatory cytokine ${ }^{[26]}$. Different from the functions of IL-1 $\beta$ and astrocytes in the inflammatory reaction, eNOS can attenuate inflammation and subsequent disease progression. In the present study, we found that chronic administration of CGD significantly inhibited IL-1 $\beta$ expression and age-related astrogliosis and astrocytosis. At the same time, CGD significantly increased eNOS expression in hippocampal CA1 and CA2/3 regions of aged rats. Therefore, CGD might also improve learning and memory ability of the aged rats by acting as an anti-inflammatory agent. However, it is worth mentioning that CGD-induced eNOS expression in the hippocampal CA3 was upregulated much more significantly than other regions, and there is no reasonable explanation for it based on our present results and related references.

Many studies have provided evidence indicating that activation of the NO/cGMP/cGK/CREB pathway is involved in hippocampal plasticity and LTP (long term potentiation), and eNOS, rather than $\mathrm{nNOS}$, is the primary source of $\mathrm{NO}$ in the postsynaptic neuron during LTP [27]. Besides that, cAMP/PKA/ERK/CREB signaling is crucial for learning and memory [28]. So it seemed that CGD is able to enhance memory through several special cell signal transduction pathways. Behavioral analyses of animals with altered ERK signaling have revealed a crucial involvement of this cascade in learning and memory [29], and it has been shown that ERK activity was decreased in dentate gyrus of aged rats which did 
not sustain LTP [30]. Previous studies reported impaired CREB phosphorylation in the brains of $\mathrm{AD}$ patients [31]. Therefore, we investigated the effect of CGD treatment on ERK/CREB signaling. As a result, CGD significantly increased ERK and CREB phosphorylation in the hippocampus, which suggests that the learning and memory-enhancing effects of CGD might be associated with the ERK/CREB pathway.

In summary, our results suggest that CGD could improve learning and memory ability of intrinsic aging rats. The possible mechanisms are as following: first, CGD was able to improve the function of the cholinergic neurons in the hippocampus, second, it might also improve learning and memory ability of the aged rats by acting as an anti-inflammatory agent, and then, as a naturally produced drug, the learning and memory-enhancing effects of CGD might be associated with the ERK/CREB signalling. Even though our present study was exclusively based on animal testing, it proved our initial theory of the drug. Post-treatment studies will be performed in the near future to further advance our knowledge of the clinical application of CGD. In addition, why CGD-induced eNOS and TrkA expression change dramatically in different hippocampal regions of the aged rats requires further investigation.

\section{Acknowledgement}

This work was supported by a grant from Nikken Sohonsha Corporation of Japan. Coccomyxa gloeobotrydiformis was supplied by Nikken Sohonsha Corporation.

\section{Conflict of interest}

None of the authors has any conflicts of interest with regards to this research.

\section{References}

1. Hagiwara Y1, Imai T, Yamada K, Sakurai K, Atsumi C, Tsuruoka A, Mizukami H, Sasaki N, Akiyama H, Hasegawa Y. Impact of Life and Family Background on Delayed Presentation to Hospital in Acute Stroke. J Stroke Cerebrovasc Dis. 2013; S1052-3057(13):00201-2.

2. Administration on Aging. A Profile of Older Americans, Vol June 18. Department of Health and Human Services Retrieved. Washington DC. 2012.

3. [Internet] CNCA China's population aging ahead of modernization (2013 April), p20. http://www.cncaprc.gov.cn/news/26081.jhtml

4. Feher J, Kovacs I, Artico M, Cavallotti C, Papale A, Balacco Gabrieli C. Mitochondrial alterations of retinal pigment epithelium in age-related macular degeneration. Neurobiol Aging. 2006; 27: 983-93.

5. Finkel T, Holbrook NJ. Oxidants, oxidative stress and the biology of ageing. Nature. 2000; 208: 239- 47.

6. Gianni P, Jan KJ, Douglas MJ, Stuart PM, Tarnopolsky MA. Oxidative stress and the mitochondrial theory of aging in human skeletal muscle. Exp Gerontol. 2004; 39:1391-400.

7. Devall M1, Mill J, Lunnon K. The mitochondrial epigenome: a role in Alzheimer's disease? Epigenomics. 2014; 6(6):665-75.

8. Franceschi C, Capri M, Monti D, Giunta S, Olivieri F, Sevini F, Panourgia MP, Invidia L, Celani L, Scurti M, Cevenini E, Castellani GC, Salvioli S. Inflammaging and anti-inflammaging: a systemic perspective on aging and longevity emerged from studies in humans. Mech Age Dev. 2007; 128: 92-105.

9. Martin DS, Lonergan PE, Boland B, Fogarty MP, Brady M, Horrobin DF, Campbell VA, Lynch MA. Apoptotic changes in the aged brain are triggered by interleukin-1beta-induced activation of $\mathrm{p} 38$ and reversed by treatment with eicosapentaenoic acid. J Biol Chem. 2002; 277(37): 34239-46.
10. Krysko DV, Agostimis P, Krysko O, Garg AD, Bachert C, Lambrecht BN, Vandenabeele P. Emerging role of damage-associated molecular patterns derived from mitochondria in inflammation. Trends Immunol. 2011; 32: 157-64.

11. Haddad JJ. Glutathione depletion is associated with augmenting a proinflammatory signal: evidence for an antioxidant/pro-oxidant mechanism regulating cytokines in the alveolar epithelium. Cytokines Cell Mol Ther. 2000; 6: 177-87.

12. Sun L, Jin Y, Dong L, Sumi R, Jahan R, Li Z. The Neuroprotective Effects of Coccomyxa Gloeobotrydiformis on the Ischemic Stroke in a Rat Model. Int J Biol Sci. 2013; 9(8):811-7.

13. Carey AN, Fisher DR, Joseph JA, Shukitt-Hale B. The ability of walnut extract and fatty acids to protect against the deleterious effects of oxidative stress and inflammation in hippocampal cells. Nutr Neurosci. 2013; 16(1):13-20

14. Bartus RT, Dean RL 3rd, Beer B, Lippa AS. The cholinergic hypothesis of geriatric memory dysfunction. Science. 1982; 217(4558): 408-14.

15. Toliver-Kinsky T1, Wood T, Perez-Polo JR. Nuclear factor kappaB/p49 is a negative regulatory factor in nerve growth factor-induced cholineacetyltransferase promoter activity in PC12 cells. J Neurochem. 2000; 75(6):2241-51.

16. Ginsberg SD1, Che S, Wuu J, Counts SE, Mufson EJ. Down regulation of trk but not p75NTR gene expression in single cholinergic basal forebrain neurons mark the progression of Alzheimer's disease. J Neurochem. 2006; 97(2): 475-87.

17. Ziesché E1, Bachmann M, Kleinert H, Pfeilschifter J, Mühl H. The interleukin-22/STAT3 pathway potentiates expression of inducible nitric-oxide synthase in human colon carcinoma cells. J Biol Chem. 2007; 282(22): 16006-15.

18. Lei $Y 1$, Chen J, Zhang W, Fu W, Wu G, Wei H, Wang Q, Ruan J. In vivo investigation on the potential of galangin, kaempferol and myricetin for protection of D-galactose-induced cognitive impairment. Food Chem. 2012; 135(4):2702-7.

19. Erickson CA, Barnes CA. The neurobiology of memory changes in normal aging. Exp Gerontol. 2003; 38(1-2):61-9.

20. Bishop NA, Lu T, Yankner BA. Neural mechanisms of ageing and cognitive decline. Nature. 2010; 464:529-35.

21. Niewiadomska G, Komorowski S, Baksalerska-Pazera M. Amelioration of cholinergic neurons dysfunction in aged rats depends on the continuous supply of NGF. Neurobiol Aging. 2002; 23(4): 601-13.

22. Saragovi HU. Progression of age-associated cognitive impairment correlates with quantitative and qualitative loss of TrkAreceptor protein in nucleus basalis and cortex. J Neurochem. 2005; 95(5):1472-80.

23. Shing YL1, Rodrigue KM, Kennedy KM, Fandakova Y, Bodammer N, Werkle-Bergner M, Lindenberger U, Raz N. Hippocampal subfield volumes: age, vascular risk, and correlation with associative memory. Front Aging Neurosci. 2011; 3:2.

24. Maher FO, Martin DS, Lynch MA. Increased IL-1beta in cortex of aged rats is accompanied by downregulation of ERK and PI-3 kinase. Neurobiol Aging. 2004; 25(6): 795-806.

25. Hu S, Peterson PK, Chao CC. Cytokine-mediated neuronal apoptosis. Neurochem Int .1997; 30(4-5):427-31.

26. Cowley TR, O'Sullivan J, Blau C, Deighan BF, Jones R, Kerskens C, Richardson JC, Virley D, Upton N, Lynch MA. Rosiglitazone attenuates the age-related changes in astrocytosis and the deficit in LTP. Neurobiol Aging. 2012; 33(1):162-75

27. Antonova I, Arancio O, Trillat AC, Wang HG, Zablow L, Udo H, Kandel ER, Hawkins RD. Rapid increase in clusters of presynaptic proteins at onset of long-lasting potentiation. Science. 2001; 294(5546): 1547-1550.

28. Kawahata I1, Yoshida M, Sun W, Nakajima A, Lai Y, Osaka N, Matsuzaki K, Yokosuka A, Mimaki Y, Naganuma A, Tomioka Y, Yamakuni T. Potent activity of nobiletin-rich Citrus reticulata peel extract to facilitate cAMP/PKA/ERK/CREB signaling associated with learning and memory in cultured hippocampal neurons: identification of the substances responsible for the pharmacological action. J Neural Transm. 2013; 120(10):1397-409

29. Atkins CM, Selcher JC, Petraitis JJ, Trzaskos JM, Sweatt JD. The MAPK cascade is required for mammalian associative learning. Nat Neurosci. 1998; 1(7):602-9.

30. McGahon B, Maguire C, Kelly A, Lynch MA. Activation of p42 mitogen-activated protein kinase by arachidonic acid and trans-1-amino-cyclopentyl-1,3- dicarboxylate impacts on long-term potentiation in the dentate gyrus in the rat: analysis of age-related changes. Neuroscience. 1999; 90(4):1167-75.

31. Yamamoto-Sasaki M, Ozawa H, Saito T, Rosler M, Riederer P . Impaired phosphorylation of cyclic AMP response element binding protein in the hippocampus of dementia of the Alzheimer type. Brain Res. 1999; 824: 300-3. 